\title{
Statistical investigations on nitrogen-vacancy center creation
}

D. Antonov, T. Häußermann, A. Aird, J. Roth, H.-R. Trebin, C. Müller, L. McGuinness, F. Jelezko, T. Yamamoto, J. Isoya, S. Pezzagna, J. Meijer, and J. Wrachtrup

Citation: Appl. Phys. Lett. 104, 012105 (2014); doi: 10.1063/1.4860997

View online: https://doi.org/10.1063/1.4860997

View Table of Contents: http://aip.scitation.org/toc/apl/104/1

Published by the American Institute of Physics

\section{Articles you may be interested in}

Engineering shallow spins in diamond with nitrogen delta-doping Applied Physics Letters 101, 082413 (2012); 10.1063/1.4748280

Nanoscale nuclear magnetic resonance with a 1.9-nm-deep nitrogen-vacancy sensor Applied Physics Letters 104, 033102 (2014); 10.1063/1.4862749

Effect of low-damage inductively coupled plasma on shallow nitrogen-vacancy centers in diamond Applied Physics Letters 107, 073107 (2015); 10.1063/1.4929356

Conversion of neutral nitrogen-vacancy centers to negatively charged nitrogen-vacancy centers through selective oxidation

Applied Physics Letters 96, 121907 (2010); 10.1063/1.3364135

Generation of single color centers by focused nitrogen implantation

Applied Physics Letters 87, 261909 (2005); 10.1063/1.2103389

Implantation of labelled single nitrogen vacancy centers in diamond using ${ }^{15} \mathrm{~N}$

Applied Physics Letters 88, 023113 (2006); 10.1063/1.2158700

\section{COMSOL} CONFERENCE 2018 BOSTON 


\title{
Statistical investigations on nitrogen-vacancy center creation
}

\author{
D. Antonov, ${ }^{1, a)}$ T. Häußermann, ${ }^{1}$ A. Aird, ${ }^{1}$ J. Roth, ${ }^{2}$ H.-R. Trebin, ${ }^{2}$ C. Müller, ${ }^{3}$ \\ L. McGuinness, ${ }^{3}$ F. Jelezko, ${ }^{3}$ T. Yamamoto, ${ }^{4}$ J. Isoya, ${ }^{5}$ S. Pezzagna, ${ }^{6}$ J. Meijer, ${ }^{6}$ \\ and J. Wrachtrup ${ }^{1}$ \\ 13. Physikalisches Institut, Universität Stuttgart, Pfaffenwaldring 57, 70569 Stuttgart, Germany \\ ${ }^{2}$ Institut für Theoretische und Angewandte Physik, Universität Stuttgart, Pfaffenwaldring 57, 70569 Stuttgart, \\ Germany \\ ${ }^{3}$ Institut für Quantenoptik, Universität Ulm, Albert-Einstein-Allee 11, 89081 Ulm, Germany \\ ${ }^{4}$ Japan Atomic Energy Agency, Takasaki, Gunma 370-1292, Japan \\ ${ }^{5}$ Graduate School of Library, Information and Media Studies, University of Tsukuba, 1-2 Kasuga, Tsukuba, \\ Ibaraki 305-8550, Japan \\ ${ }^{6}$ Institut für Experimentelle Physik II, Linnéstrasse 5, 04103 Leipzig, Germany
}

(Received 7 November 2013; accepted 17 December 2013; published online 2 January 2014)

\begin{abstract}
Quantum information technologies require networks of interacting defect bits. Color centers, especially the nitrogen vacancy $\left(\mathrm{NV}^{-}\right)$center in diamond, represent one promising avenue, toward the realisation of such devices. The most successful technique for creating $\mathrm{NV}^{-}$in diamond is ion implantation followed by annealing. Previous experiments have shown that shallow nitrogen implantation $(<10 \mathrm{keV})$ results in $\mathrm{NV}^{-}$centers with a yield of $0.01 \%-0.1 \%$. We investigate the influence of channeling effects during shallow implantation and statistical diffusion of vacancies using molecular dynamics and Monte Carlo simulation techniques. Energy barriers for the diffusion process were calculated using density functional theory. Our simulations show that $25 \%$ of the implanted nitrogens form a NV center, which is in good agreement with our experimental findings. ( 2014 AIP Publishing LLC. [http://dx.doi.org/10.1063/1.4860997]
\end{abstract}

A basic requirement for applications of solid-state defects in quantum information processing (QIP), magnetometry, and even for biomarkers is the accurate placement of impurities in the target material. A promising candidate for these applications is the negatively charged nitrogenvacancy $\left(\mathrm{NV}^{-}\right)$center embedded in diamond. The defect consists of a carbon $(\mathrm{C})$ atom which is replaced by a nitrogen $(\mathrm{N})$ atom together with an adjacent vacancy $(\mathrm{V})$ and an additional electron captured from the diamond lattice.

Experimentally, ion implantation techniques are used to introduce $\mathrm{N}$ and $\mathrm{V}$ defects in the carbon matrix. Upon annealing the diamond above $750 \mathrm{~K}$ (Ref. 1) for several hours, the fixed $\mathrm{N}$ atoms capture mobile $\mathrm{V}$ creating $\mathrm{NV}$ centers. Experiments have shown that shallow nitrogen implantations $(<10 \mathrm{keV})$ result in a $\mathrm{NV}^{-}$yield of $0.01 \%-0.1 \%{ }^{2}$ while implantation at higher energies results in a NV center yield of $45 \%{ }^{2}$ To resolve this apparent discrepancy, we carried out molecular dynamics (MD) investigations of the implantation and annealing process at low energy.

Specifically, we present simulation results of NV center creation. We study the statistical influence of channeling effects $^{3}$ on shallow implantations. Further we focus on the statistical diffusion of vacancies in diamond and its impact on the NV center yield. Our simulations show a significant difference in the NV yield compared with the earlier experiments. Simulations suggest that $25 \%$ of the implanted nitrogens form a NV center after annealing. Additionally, we compare to experimental results of shallow implantation in the range of $2.5-20 \mathrm{keV}$. The experimental results are in good agreement with the simulation.

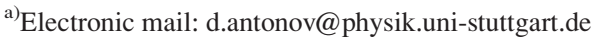

Using the ITAP MD (IMD) Program, ${ }^{4,5}$ a diamond system of $5.5 \times 5.5 \times 52 \mathrm{~nm}^{3}$ was used for simulations; the C-C and N-C interactions were represented using a Tersoff potential $^{6-10}$ with interaction parameters from Refs. 11 and 12. For decreasing $\mathrm{C}-\mathrm{C}$ or $\mathrm{N}-\mathrm{C}$ distances, the potential energy of the Tersoff potential reaches a constant limiting value, allowing the atoms to move arbitrarily close to one other. Therefore, an additional Ziegler-Biersack-Littmark (ZBL) potential was added. ${ }^{13,14}$ For atom separations $r<0.3 \AA$, the Tersoff potential is smoothly switched to the ZBL potential.

In order to bring a system into equilibrium at room temperature, a thermal cycle was employed. It consists of five NPT (number of particles, pressure, and temperature are conserved) and one final NVE (number of particles, volume, and pressure are conserved) ensemble simulation step. The first four are used to heat the structure up to $1000 \mathrm{~K}$, relax it at $1000 \mathrm{~K}$, cool it down to room temperature (RT), and to relax the diamond at RT using periodic boundaries in all directions. In the last NPT step, the periodic boundary conditions in the $z$ direction (implantation direction) are removed. The final NVE simulation equilibrates the system with the ground layer of the diamond fixed in the $z$ direction.

All NPT ensemble simulations have been done with a time step of $0.1 \mathrm{fs}$ and the NVE equilibration with time steps of $0.01 \mathrm{fs}$. The implantation process itself was simulated by adding the impact nitrogen atom on top of the diamond surface ((100) direction), at a distance $d=2.51 \AA$ above the surface; this distance is beyond the range of the Tersoff potential. The particle is then implanted on a normal incident trajectory with kinetic energy $E_{k}=4 \mathrm{keV}$.

We analyse the structure after implantation by comparing the damaged structure with the initial structure before 
implantation and distinguish between vacancy, lattice atom, and complex structures. We also investigate the influence of impact atom parameters on the following quantity: Depth of the impact atom, displacement in $x y$-direction (straggling), and kinetic energy of the impact atom.

To get qualitative insight into our MD simulation, we compare Crystal-TRIM (CTRIM) ${ }^{15}$ simulations to MD results. The CTRIM method uses a ZBL potential for atom interactions and includes an electronic stopping power which is missing in the MD simulations. The results are discussed later in the paper.

To investigate the probability for NV center creation during annealing, we also conducted kinetic Monte-Carlo (MC) simulations based on the simple hopping frequency of defects

$$
\Gamma=\omega_{a} e^{-\frac{E_{a}}{k_{B} T}} .
$$

Here, $\omega_{a}=10^{13} \mathrm{~s}^{-1}$ denotes the attempt frequency with which defects attempt to overcome a barrier of energy $E_{a}$. The values for activation energies are $2.3 \mathrm{eV}$ for vacancy $(\mathrm{V}),{ }^{1,16} 1.7 \mathrm{eV}$ for interstitial nitrogen $\left(\mathrm{N}_{I}\right),{ }^{17}$ and 1.5 for carbon interstitial $\left(\mathrm{C}_{I}\right) \cdot{ }^{18}$

Additionally, density functional theory (DFT) simulations using the ABINIT ${ }^{19-21}$ package have been conducted to investigate a possible uphill potential a vacancy has to pass when approaching a nitrogen atom. Such a potential would considerably reduce the likelihood of NV center formation. For the calculations, the projector augmented wave (PAW) method and a $2 \times 2 \times 2$ Monkhorst-Pack grid were used for k-point sampling. The exchange correlation potential $\left(V_{x c}\right)$ was approximated by local density approximation (LDA). The examined structures, including second, third, fourth, and sixth next neighbour configurations of an on-site nitrogen and a vacancy showed the same total energy within errors. The only exception is a specific configuration with a vacancy on the most distant third next neighbor positions (Figure 1). With a nitrogen on lattice site A, it is energetically more favorable for a vacancy to be at the third

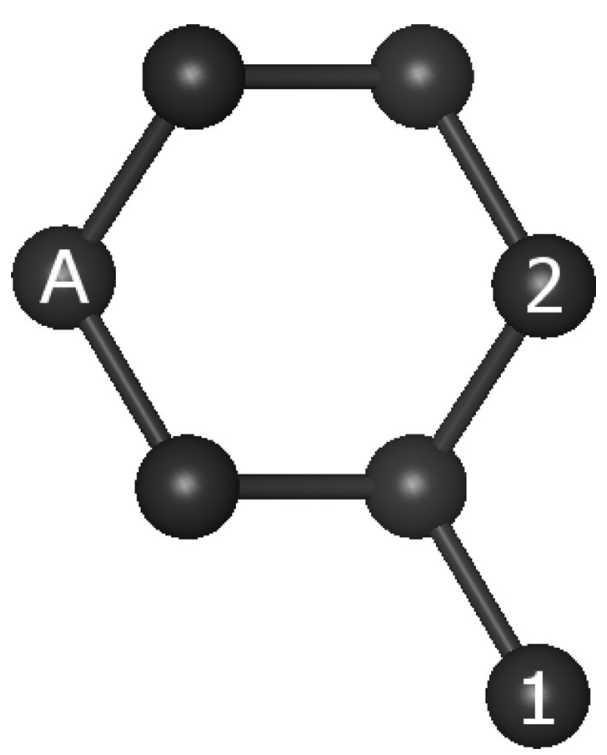

FIG. 1. Two different atom configurations of $\mathrm{N}$ (labeled A) and V (labeled 1 or 2) in the diamond matrix. next-neighbor site labeled 1 instead of 2 . This is due to symmetry reasons, since the lattice is able to relax which decreases the total energy by about $0.7 \mathrm{eV}$. Except of the above mentioned case, no evidence for an uphill potential in vacancy diffusion could be found. To simulate annealing for implantation close to the diamond surface, we considered defect structures of different implantation energies. Since IMD does not take the electronic stopping into account, only the $4 \mathrm{keV}$ defect structure is simulated by this method. For higher implantation energies, defect structures simulated by the Stopping and Range of Ions in Matter (SRIM) ${ }^{13}$ $(5-50 \mathrm{keV})$ are used. The crystal size chosen in the simulations corresponds to a final concentration of about $1 \times$ $10^{17} \mathrm{~cm}^{-3}$ nitrogen atoms by taking into account the distribution in stopping depth. A simulated time of $2 \mathrm{~h}$ of annealing at $1100 \mathrm{~K}$ decreases the dynamics of vacancies within the crystal such that only a negligible number $(<5 \%)$ are still mobile the end of the simulation.

In recent years, near-surface impurities, in particular, $\mathrm{NV}$ centers in diamond, have been extensively studied experimentally. Magnetic field imaging, ${ }^{22}$ fluorescence resonance energy transfer (FRET) ${ }^{23}$ and nuclear magnetic resonance $(\mathrm{NMR})^{24}$ using $\mathrm{NV}^{-}$centers in diamond rely on the weak magnetic dipole interaction which decreases with the distance by $1 / r^{3}$. Accordingly, sensing applications require reliable production of shallow $\mathrm{NV}^{-}$.

Several studies have shown a significant influence of channeling effects on the depth distribution of implanted nitrogens in diamond giving a larger than expected implantation depth. ${ }^{25}$ Usual methods, such as SRIM, underestimate the defect depth by a factor of two, ${ }^{25}$ as SRIM assumes an amorphous structure for diamond. We study implantation processes using IMD and CTRIM, which take the crystal structure into account.

Figure 2 shows the depth distribution of $4 \mathrm{keV}$ nitrogen implantation along (100) direction. The time demanding MD simulations were repeated 120 times to provide statistics, in each of which the $\mathrm{N}$ starting position was varied in order to get statistical average values for the depth distribution. The results of MD simulations were compared to CTRIM simulations (Figure 3). A broad distribution can be extracted from

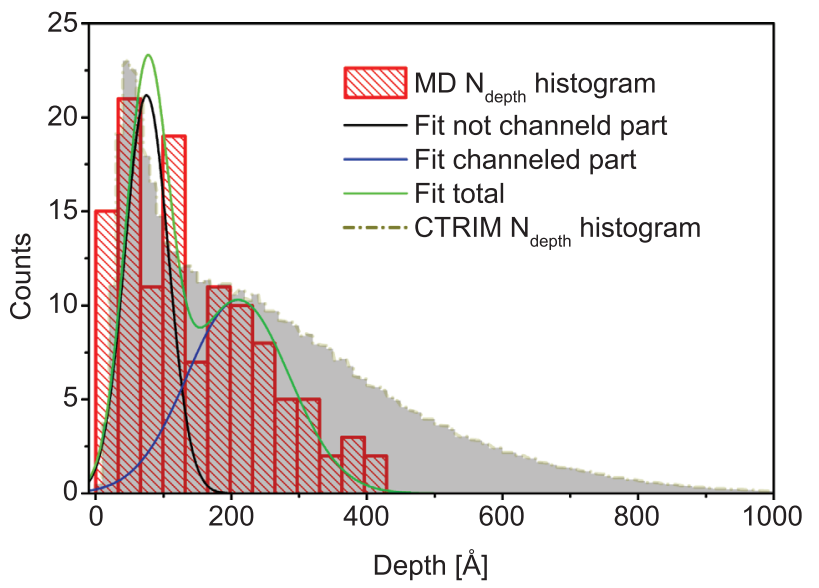

FIG. 2. Depth distribution of single implanted nitrogen atoms with an incident angle of $0^{\circ}$ and with an implantation energy of $4 \mathrm{keV}$; red: MD simulation, grey: CTRIM simulation. The black and blue curves are Gaussian fits summarized in a cumulative curve (green). 


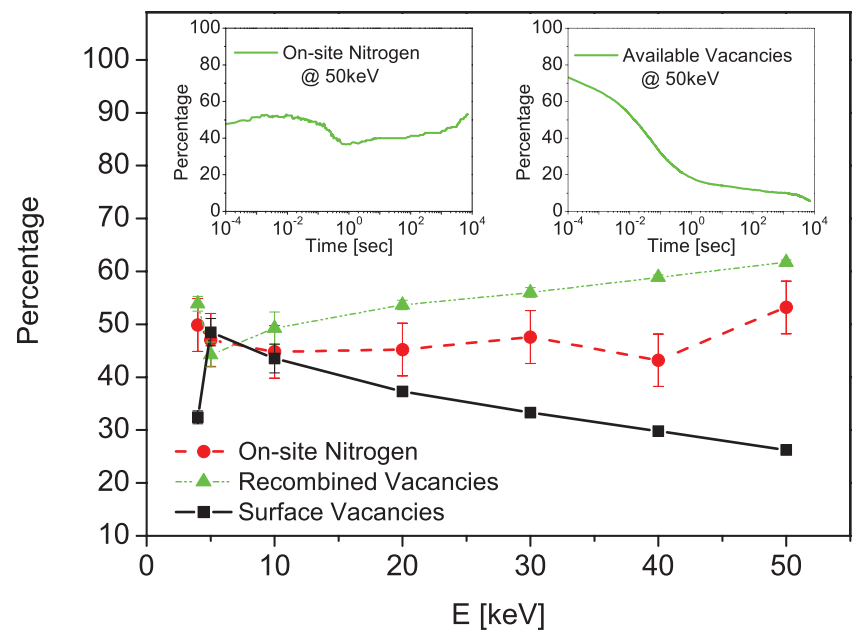

FIG. 3. Number of on-site nitrogens (circles) and number of vacancies which are lost to the surface (squares) or lost due to recombination processes (triangles) after $2 \mathrm{~h}$ of simulated annealing depending on the implantation energy. The left inset shows the time evolution of on-site nitrogens during annealing after $50 \mathrm{keV}$ implantations with an initial $40 \%$ on-site and $60 \%$ interstitial distribution. The number of on-site nitrogen atoms increases already within the first simulation steps which are not shown. The right inset shows the number of vacancies that are still available for NV center creation over time after $50 \mathrm{keV}$ implantations.

simulation resulting from a combination of two processes, a "un-channeled"-at lower depth, and a "channeled"-at larger depth. In MD simulations, nitrogens are implanted at a depth of $0-460 \AA$. The data can be fitted using two Gaussian peaks at $d_{c 1}=75 \AA$ (first peak: black curve) and $d_{c 2}=210 \AA$ (second peak: blue curve). The first Gaussian peak has a FWHM of $77 \AA$, whereas the second Gaussian peak has a FWHM of $177 \AA$. The number of implanted nitrogen atoms at $d_{c 1}$ is 21 and at $d_{c 2} 10$. In addition, the simulations show that $37 \%$ of implanted nitrogens end up on a lattice site and $63 \%$ end up as interstitials.

The CTRIM results (Figure 2) differ from the MD outcome. Here, the number of projectiles was chosen to be $10^{6}$; the CTRIM results are scaled in order to make them comparable with MD results. The "un-channeled" part has a $25 \%$ narrower and the "channeled" part a $33 \%$ broader distribution than in MD simulation. The maximum of the "un-channeled" peak is shifted by $25 \AA$ (33\%) towards lower implantation depth, whereas the maximum of the "channeled" peak is shifted by $20 \AA(10 \%)$ towards higher implantation depth compared to MD. This might be due to the fact that during implantation a higher number of nitrogens enter the crystal, i.e., in CTRIM, one implants into the pre damaged lattice. Additionally, the empirical potential used in both methods give rise to different results. The bond ordered Tersoff potential used in MD describes the interaction between the projectile and the diamond atoms more accurately than the ZBL potential used in CTRIM. This leads to a higher number of channeled projectiles.

After implantation, it is unlikely to find a next nearest neighbor pair of a nitrogen atom and a vacancy. An annealing step is required such that vacancies become mobile and diffuse through the lattice. Several studies on diffusion near to the surface have been done in recent years. ${ }^{26-29}$ Here, we model the diffusion process during annealing and investigated its influence on the NV center yield.
The statistical probability for vacancies, created during implantation, to migrate to a next neighbor position of the implanted nitrogen was examined by Monte Carlo simulations. Our analysis included the most important loss mechanisms for vacancies, such as migration to the surface or recombination with interstitials. The processes, in which a self-interstitial atom replaces an on-site nitrogen at a lattice site and turns the latter into an interstitial nitrogen atom, ${ }^{16}$ were also included. In contrast to on-site nitrogen atoms which hardly move at $1100 \mathrm{~K}$, interstitial nitrogen atoms are mobile during annealing. ${ }^{16,30}$ The initial distribution after implantation was assumed to be $40 \%$ on-site nitrogen and $60 \%$ between lattice sites, as suggested by the MD simulations discussed above. 100 annealing runs with different starting configurations were performed. Fig. 3 shows the percentage of nitrogen atoms that are found on lattice sites after annealing. The number is almost identical for all energies within error bars. Hence, only about half of the implanted nitrogen atoms are available for NV center creation in the first place. This is in good accordance with the maximum yield found by Pezzagna et al. ${ }^{2}$ for $\mathrm{MeV}$ implantations. It suggests that for high energies every implanted nitrogen that finally ends up on a lattice site is turned into a $\mathrm{NV}^{-}$center.

Figure 3 shows the percentage of initially created vacancies that are lost at the surface or due to recombination. By considering initial SRIM input $(5-50 \mathrm{keV})$, as expected, at shallow implantations, more vacancies $(48 \%)$ are lost at the surface than deep ones inside the lattice (26\%). This is because of the short distance to the surface that makes it more likely for vacancies and self-interstitials to randomly migrate to the surface where they are lost, instead of recombining. Except for very low implantation energies $(<20 \mathrm{keV})$, the loss due to recombination with interstitial carbon is significantly higher. It increases from $44 \%$ at $5 \mathrm{keV}$ to $62 \%$ at $50 \mathrm{keV}$, while the loss at the surface decreases from $49 \%$ to $26 \%$. The line crossing in Figure 3 of the vacancies lost at the surface and due to recombination defines a threshold; for implantation, energies lower than $7 \mathrm{keV}$ loss of vacancies is dominated by the surface process and for higher implantation, energies recombination process reduces the available vacancies to form NV centers. The simulations show that the recombination of vacancies and interstitials happens within the first seconds of annealing because of the high mobility of carbon interstitials. Hence, the number of vacancies that are left to possibly migrate towards a nitrogen atom is less than half the number that is suggested by implantation simulations such as SRIM, which does not take recombination into account. The results at $4 \mathrm{keV}$ implantation energy based on IMD implantation simulations show an even higher probability of recombination than the results based on SRIM data. IMD seems to yield defect structures with smaller distances between vacancies and the corresponding interstitials which therefore are more likely to recombine. The right inset in Figure 3 shows the percentage of remaining vacancies that are still available for NV center creation after $50 \mathrm{keV}$ implantations. It can be seen that at the end of the simulation the vacancy diffusion within the crystal has almost completely come to an end. On average $90 \%$ of the created vacancies during the implantation process are lost after $2 \mathrm{~h}$ of annealing. 


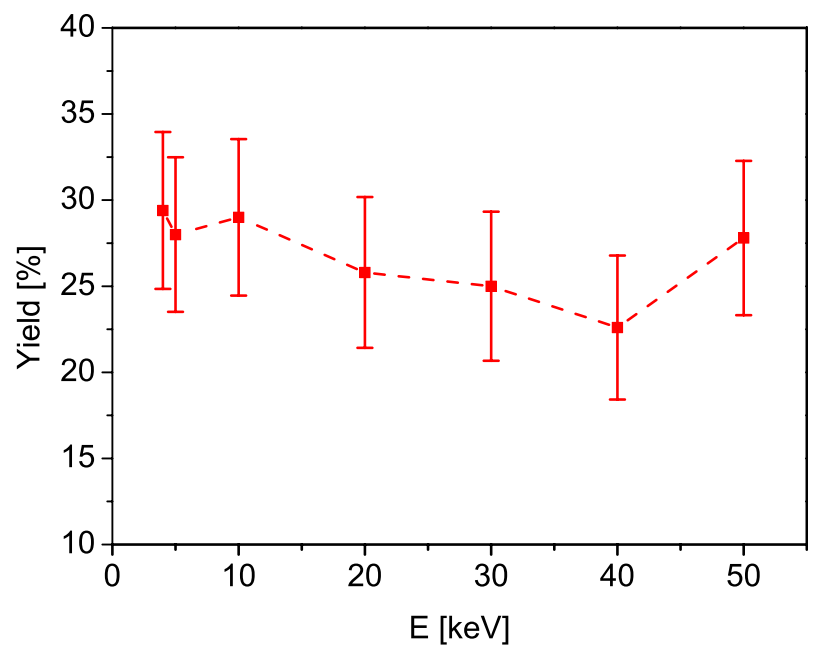

FIG. 4. Number of NV centers created after implantation and annealing.

Summarising the result of implantation and annealing simulations, Figure 4 shows the yield of NV centers of any charge state that is to be expected. It is about the same for all investigated implantation energies roughly $25 \%$ of implanted ions are expected to yield a NV center. Implantations experiments were done to experimentally verify the simulation results.

The characterisation of the shallow NV centers was performed on a custom built confocal microscope at room temperature. High vacuum is essential to avoid etching of the first diamond layers leading to a lower observed yield. After annealing, the diamonds were boiling in a mixture of sulfuric, nitric and perchloric acids for several hours at $180^{\circ} \mathrm{C}$, in order to give a heterogeneous oxygen terminated diamond surface. This surface treatment is known to allow observation of $\mathrm{NV}^{-}$centers at low depths.

Confocal images of the diamond surfaces after the implantation process were taken. In each image, the total number of created NVs, consisting of $\mathrm{NV}^{-}$and $\mathrm{NV}^{0}$, of an area of $15 \mu \mathrm{m} \times 15 \mu \mathrm{m}$ was counted and the yield calculated. The results are given in Table I. The data show that the number of created NV centers depends on the implantation energy and on the implantation dose. The yield of the total number of NV centers increases just slightly for a dose of $3 \times 10^{8}$ ions $/ \mathrm{cm}^{2}$ with implementation energy, resulting in $23 \%-29 \%$. No change in yield $(\sim 20 \%)$ for higher implantation energies $(20 \mathrm{keV}, 30 \mathrm{keV})$ with similar implantation dose is observed.

In this paper, we present results using MD, DFT, and MC simulation techniques to investigate the NV center yield after nitrogen implantation in diamond. Additionally we show experimental data and compare them to these simulations. We could demonstrate that during nitrogen implantations into

TABLE I. NV yield depending on implantation energy and doses.

\begin{tabular}{lcc}
\hline \hline Implantation energy $[\mathrm{keV}]$ & Doses $\left[\right.$ ions $\left./ \mathrm{cm}^{2}\right]$ & Yield $[\%]$ \\
\hline 2.5 & $3 \times 10^{8}$ & 23 \\
5 & $3 \times 10^{8}$ & 29 \\
$20^{\mathrm{a}}$ & $5 \times 10^{7}$ & 21 \\
$30^{\mathrm{a}, \mathrm{b}}$ & $2 \times 10^{8}$ & 20 \\
\hline \hline
\end{tabular}

${ }^{\text {aD }}$ ata are performed with ${ }^{15} \mathrm{~N}_{2}^{+}$which share the implantation energy. For all the other implantations, ${ }^{15} \mathrm{~N}^{+}$is used.

${ }^{\mathrm{b}}$ Data are taken from Ref. 31. diamond even at low implantation energies $(4 \mathrm{keV})$ channeling effects occur and have to be taken into account for accurate placement in the carbon matrix. Furthermore, annealing of the implanted diamond structures leads to a final NV center yield of about $25 \%$. This result is significantly higher than the $\mathrm{NV}^{-}$ yield seen in earlier experimental publications $(0.01 \%-0.1 \%)^{2}$ with implantation energies $<10 \mathrm{keV}$. The discrepancy can be explained by the charge state of the shallow implanted NV centers or etching of the diamond during the annealing process. Experimentally only the $\mathrm{NV}^{-}$and the $\mathrm{NV}^{0}$ can be detected. Surface effects or damage structures in the vicinity of the NV center could change the charge of the $\mathrm{NV}^{-}$; leaving the $\mathrm{NV}^{+}$ state which has not been detected yet. Experimentally, a maximum $\mathrm{NV}$ yield $\left(\mathrm{NV}^{-}\right.$and $\left.\mathrm{NV}^{0}\right)$ up to $29 \%$ for implantation energies in a range of $2.5 \mathrm{keV}-20 \mathrm{keV}$ was found. This is in a good agreement with our simulation results. At this point, we have to mention that we observed a decrease in the yield for an implantation energy of $5 \mathrm{keV}$ by a factor of 3 by increasing the dose by one magnitude. This effect enhances by lowering the implantation energy or increasing the dose. An explanation could be found in the damaged region in the vicinity of the implanted nitrogen which does not recover during the annealing process. Saada et $a l .{ }^{32}$ and Sorkin $e t a l .{ }^{33}$ investigated the annealing behaviour of diamond using MD simulations. They observed that a damaged region with $60 \% \mathrm{sp}^{2}$-bonded carbon atoms form a graphitic geometry after annealing. Graphitisation may render fluorescence of NV centers undetectable, e.g., by changing the charge state to $\mathrm{NV}^{+}$. For lower implantation dose or higher implantation energy, the damage in the vicinity of the NV defect is reduced. After the annealing, the NV center is surrounded by a diamond like structure; leaving the NV detectable. Note, the simulation scheme does not consider charges and therefore give an average value for $\mathrm{NV}$ center creation of all charge states. Introducing additional electrons to the system which can be captured by the NV centers to form the $\mathrm{NV}^{-}$or carbon coimplantations ${ }^{31}$ may potentially increase the very low experimental yield.

Further investigations on the implantation and the annealing process are necessary. The IMD simulation package considers only the nuclear stopping - a valid approximation for the low energy implantations-whereas, the electronic stopping is not taken into account. In addition, the $\mathrm{NV}$ yield depending on the annealing temperature, the intrinsic nitrogen content, and the implantation densities have yet to be studied. In particular, efficient simulations for the implantation with higher energies require simulations of larger systems which are out of reach with current codes and computing powers. Adding precise calculations of the electronic states of defect structures would yield a comprehensive picture of the defect center and its surrounding necessary for the understanding of the generation process of larger spin arrays.

The authors would like to acknowledge financial support by the DFG via the SFB 716 as well as the Forschergruppe FOR 1493.

${ }^{1}$ G. Davies, S. C. Lawson, A. T. Collins, A. Mainwood, and S. J. Sharp, Phys. Rev. B 46, 13157 (1992).

${ }^{2}$ S. Pezzagna, B. Naydenov, F. Jelezko, J. Wrachtrup, and J. Meijer, New J. Phys. 12, 065017 (2010). 
${ }^{3}$ G. Hobler, Radiation Effects and Defects in Solids 139, 21 (1996).

${ }^{4}$ J. Roth, F. Gähler, and H.-R. Trebin, Int. J. Mod. Phys. C: Computational Physics \& Physical Computation 11, 317 (2000).

${ }^{5}$ J. Stadler, R. Mikulla, and H.-R. Trebin, Int. J. Mod. Phys. C 8, 1131 (1997).

${ }^{6}$ G. C. Abell, Phys. Rev. B 31, 6184 (1985).

${ }^{7}$ J. Tersoff, Phys. Rev. B 37, 6991 (1988).

${ }^{8}$ J. Tersoff, Phys. Rev. Lett. 61, 2879 (1988).

${ }^{9}$ J. Tersoff, Phys. Rev. B 38, 9902 (1988).

${ }^{10}$ J. Tersoff, Phys. Rev. Lett. 56, 632 (1986).

${ }^{11}$ J. Tersoff, Phys. Rev. B 39, 5566 (1989).

${ }^{12}$ F. de B. Mota, J. F. Justo, and A. Fazzio, Phys. Rev. B 58, 8323 (1998).

${ }^{13}$ J. Ziegler, J. Biersack, and U. Littmark, SRIM, the Stopping and Range of Ions in Matter (Pergamon Press, 1985), Vol. 1.

${ }^{14}$ H. Chan, K. Nordlund, J. Peltola, H.-J. Gossmann, N. Ma, M. Srinivasan, F. Benistant, and L. Chan, Nucl. Instr. Meth. Phys. Res. Sec. B: Beam Interactions with Materials and Atoms 228, 240 (2005).

${ }^{15}$ M. Posselt and J. Biersack, Nucl. Instr. Meth. Phys. Res. Sec. B: Beam Interactions with Materials and Atoms 64, 706 (1992).

${ }^{16}$ A. Mainwood, Phys. Rev. B 49, 7934 (1994).

${ }^{17}$ J. P. Goss, P. R. Briddon, S. Papagiannidis, and R. Jones, Phys. Rev. B 70, 235208 (2004).

${ }^{18}$ S. J. Breuer and P. R. Briddon, Phys. Rev. B 51, 6984 (1995).

${ }^{19}$ F. Bottin, S. Leroux, A. Knyazev, and G. Zérah, Comp. Mat. Sci. 42, 329 (2008).

${ }^{20}$ M. Torrent, F. Jollet, F. Bottin, G. Zérah, and X. Gonze, Comp. Mat. Sci. 42, 337 (2008).
${ }^{21}$ X. Gonze, B. Amadon, P.-M. Anglade, J.-M. Beuken, F. Bottin, P. Boulanger, F. Bruneval, D. Caliste, R. Caracas, M. Côté et al., Comp. Phys. Commun. 180, 2582 (2009).

${ }^{22}$ S. Steinert, F. Dolde, P. Neumann, A. Aird, B. Naydenov, G. Balasubramanian, F. Jelezko, and J. Wrachtrup, Rev. Sci. Instrum. 81, 043705 (2010).

${ }^{23}$ J. Tisler, R. Reuter, A. Laemmle, F. Jelezko, G. Balasubramanian, P. R. Hemmer, F. Reinhard, and J. Wrachtrup, ACS Nano 5, 7893 (2011).

${ }^{24}$ T. Staudacher, F. Shi, S. Pezzagna, J. Meijer, J. Du, C. A. Meriles, F. Reinhard, and J. Wrachtrup, Science 339, 561 (2013).

${ }^{25}$ B. K. Ofori-Okai, S. Pezzagna, K. Chang, M. Loretz, R. Schirhagl, Y. Tao, B. A. Moores, K. Groot-Berning, J. Meijer, and C. L. Degen, Phys. Rev. B 86, 081406 (2012).

${ }^{26}$ T. Halicioglu, Thin Solid Films 228, 293 (1993).

${ }^{27}$ X. Hu, Y. Dai, R. Li, H. Shen, and X. He, Solid State Commun. 122, 45 (2002).

${ }^{28}$ R. Long, Y. Dai, L. Yu, H. Jin, and B. Huang, Appl. Surf. Sci. 254, 6478 (2008).

${ }^{29}$ J. Orwa, K. Ganesan, J. Newnham, C. Santori, P. Barclay, K. Fu, R. Beausoleil, I. Aharonovich, B. Fairchild, P. Olivero et al., Diam. Rel. Mat. 24, 6 (2012).

${ }^{30}$ J. Field, The Properties of Natural and Synthetic Diamond (Academic Press, 1992).

${ }^{31}$ B. Naydenov, V. Richter, J. Beck, M. Steiner, P. Neumann, G. Balasubramanian, J. Achard, F. Jelezko, J. Wrachtrup, and R. Kalish, Appl. Phys. Lett. 96, 163108 (2010).

${ }^{32}$ D. Saada, J. Adler, and R. Kalish, Phys. Rev. B 59, 6650 (1999).

${ }^{33}$ A. Sorkin, J. Adler, and R. Kalish, Phys. Rev. B 70, 064110 (2004). 